\title{
UNCONDITIONAL STABILIZATION OF CS and CG MESFET TRANSISTOR
}

\author{
Hany F. Hammad ${ }^{\star}$, Alois P. Freundorfer ${ }^{\star}$, and Yahia M.M. Antar» \\ Electrical and Computer Engineering Department, Queen's University, Kingston Ontario, Canada, \\ $\uparrow$ hammadh@ee.queensu.ca, freund@post.queensu.ca
}

Electrical and Computer Engineering Department, `oyal Military College of Canada, antar-y@ rmc.ca

\begin{abstract}
Feedback is used to achieve multi-band unconditional stability for GaAs MESFET transistor. Analytical formulation based on evaluating the stability parameters as a function of the transistor model elements is provided, with two methods to accurately estimate the feedback values needed for all-band unconditional stability. The technique is applied to common source and common gate configurations. Both were monolithically fabricated and tested, and very good agreement between the predicted and measured results is obtained.
\end{abstract}

\section{INTRODUCTION}

Transistor stability an important issue in amplifier design (Gonzalez [1]). Generally, the transistor is designed to be stable for a given load impedance and at a given frequency band. However, there may be situations when stability is required over a wide band, especially if load characteristics could push the transistor into instability outside the operating band. A new simple, but effective approach that makes use of a series resistive-inductive feedback network to achieve unconditional stability over a large frequency range is presented. The analysis presented here allows for exact estimation of the feedback values needed to achieve the required stability, while at the same time maintaining the highest maximum available gain. This presents advantages over previous work (Perez and Ortega [2], Niclas and Ortega [3], and Niclas et al [4]), where feedback networks were used to either neutralize or flatten the gain over specified frequency range, and were restricted to the frequency range below $18 \mathrm{GHz}$. In this work, a new application for the "resistiveinductive" feedback networks is used to achieve unconditional transistor stabilization over a very wide frequency band that extends from very low frequencies till frequencies above $40 \mathrm{GHz}$. Furthermore, the technique will be applied to both the common source and the rarely employed common gate. Analytical formulas will be provided, plus a detailed description of how the feedback network can be designed.

\section{TRANSISTOR STABILITY ANALYSIS}

Transistor stability is usually defined in terms of two parameters: the stability factor $K$ and the stability measure $B 1$. Generally, transistor stability can be categorized as one of three states

- Unconditionally stable $(K>1 \& B>0)$.

- Conditionally stable (potentially unstable) $(K<1 \& B 1>0)$.

- Unstable $(K<1 \& B<0)$.

However, both $K$ and $B l$ are usually expressed in terms of the transistor $S$-parameters. This does not give a direct insight into the contribution of the different components of the transistor model on the overall transistor performance. Hence, stability parameters relations, which are function of the transistor model 
will be more useful. In order to accomplish this, first the stability parameters were evaluated as a function of the transistor admittance $Y$-matrix, give by

$$
\begin{gathered}
K=\frac{\left(2 \operatorname{Re}\left(Y_{11}\right) \operatorname{Re}\left(Y_{22}\right)-\operatorname{Re}\left(Y_{12} Y_{21}\right)\right)}{\left|Y_{12}\right| Y_{21} \mid}>1 \\
B 1=\operatorname{Re}\left(Y_{22}\right)\left(G_{o}^{2}+\left|Y_{11}\right|^{2}\right)-\operatorname{Re}\left(Y_{11} \bar{Y}_{12} \bar{Y}_{21}\right)>0
\end{gathered}
$$

Where $Y_{i j}$ is the admittance between port $i, j$, and $G_{o}$ is the characteristics conductance of the system. Secondly, the transistor admittance $Y$-matrix was computed as a function of the transistor model (Figure 1(a)). Following these steps the transistor different model elements were accurately computed using the $Y$ matrix determined from the measured $S$-parameters. This was achieved by equating the real and imaginary parts of the calculated $Y$-matrix to the measured $Y$-matrix. Next, the transistor $Y$-matrix is used in equations (1) and (2), yielding two equations that describe $K$ and $B 1$ in terms of the MESFET transistor model elements. The procedure was applied to both the common source and common gate configurations. As a result, it was possible to gain physical insight in the transistor performance by examining the model parameters. For example, for the common source configuration it was found that the stability condition $B 1$ is satisfied for all frequency bands $(B 1>0)$ while the $K$ condition yields the following relation

$$
\omega>\frac{1}{\left(T+\frac{2 r_{g}\left(C_{g s}+C_{g d}\right)^{2}}{r_{d s} C_{g d} g_{m o}}\right)}
$$

In equation (3), $\omega$ is the angular frequency, while $r_{g}, C_{g s}, C_{g d}, r_{d s}, g_{m o}$, and $T$ represents the model components shown in Figure 1(a). The transistor is unconditionally stable as long as (3) is satisfied. Based on that, different cases were studied, and they were all compared for their corresponding stability performance and their maximum achievable stable gain $\left(G_{\max }\right)$. It was determined that a resistive inductive feedback network (Figure 1-(b)) provided the best overall performance. Finally an accurate but rather simple technique of providing the needed values of the feedback resistive $\left(R_{f}\right)$ and inductive values $\left(L_{f}\right)$ was established.

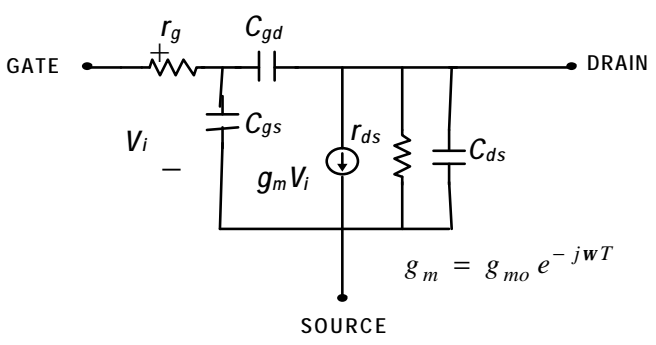

(a)

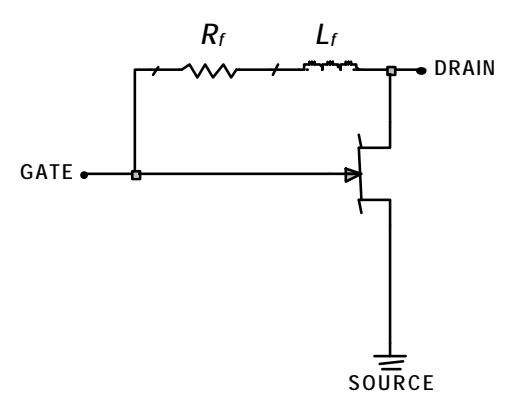

(b)

Figure 1 (a) The employed transistor model, (b) The feedback stabilization network

\section{CALCULATING THE FEEDBACK PARAMETERS}

The total admittance of the transistor with feedback is given by 


$$
Y_{\text {Total }}=\left[\begin{array}{ll}
Y_{11}+Y_{f} & Y_{12}-Y_{f} \\
Y_{21}-Y_{f} & Y_{22}+Y_{f}
\end{array}\right]
$$

Where $Y_{f}$ is the feedback admittance given by

$$
Y_{f}=\frac{1}{R_{f}+j \omega L_{f}}
$$

and $Y_{i j}$ is the admittance between port $i, j$ of the two port transistor model network. Substituting equation (4) in equation (1) and (2), yields two relations that relate of the feedback parameters to the frequency. Hence the problem reduces to finding the optimum values needed to achieve the all band unconditional stability. Two different methods were established to compute and estimate these optimum values.

In the first method, the expression for the stability factor $K$ was analytically examined and approximate formulas for calculation of the feedback parameters were obtained. The second method, which provided a check on the accuracy of the first method, is explained here. The stability and maximum stable gain contours were plotted as a function of frequency and feedback resistance for a given feedback inductance (Fig. (2)). This provides design curves that could be used for unconditional stability evaluations or for trade off between the stability and gain. Drawing a horizontal line passing by the minimum value of $K=1$ contour yields the maximum value of $R_{f}$ needed for all band conditional stability. For common source transistor with feedback inductance $L_{f}=0.1 \mathrm{nH}$, Fig. (2) shows that maximum $R_{f}$ of $592 \Omega$ is needed for all band unconditional stability, and the $G_{\max }(5.6 \mathrm{~dB})$ value that will be available at $30 \mathrm{GHz}$. Figure 3-(b), shows the $K$, and $B 1$ stability parameters for the transistor before and after the stabilization was performed with the values for stabilization obtained from Figure 3-(b), given by $L_{f}=0.1 \mathrm{nH}$ and $R_{f}=592 \Omega$. The values of $\mathrm{K}$ and B1 with feedback are both satisfied and the transistor is unconditionally stable from zero to $40 \mathrm{GHz}$, and the trends of both curves shows that the transistor could be still unconditionally stable beyond $40 \mathrm{GHz}$.

The transistor employed is a Nortel Network (NT) double-connected gate MESFET transistor with six fingers and a total width of $300 \mu \mathrm{m}$. The NT MESFET had the following parameters: $V_{p}=-1.2 \mathrm{~V}, I_{d s s}=120$ $\mathrm{A} / \mathrm{mm}, f_{t}=20 \mathrm{GHz}$ and $F_{\min }<1 \mathrm{~dB}$ up to $5 \mathrm{GHz}$. Several stabilized transistors were monolithically fabricated, and measured. The stabilized transistors were later used in building $C P W$-based (coplanar waveguide) amplifiers operating at $30 \mathrm{GHz}$. The amplifiers design and modeling procedure together with the measured results will be presented separately.
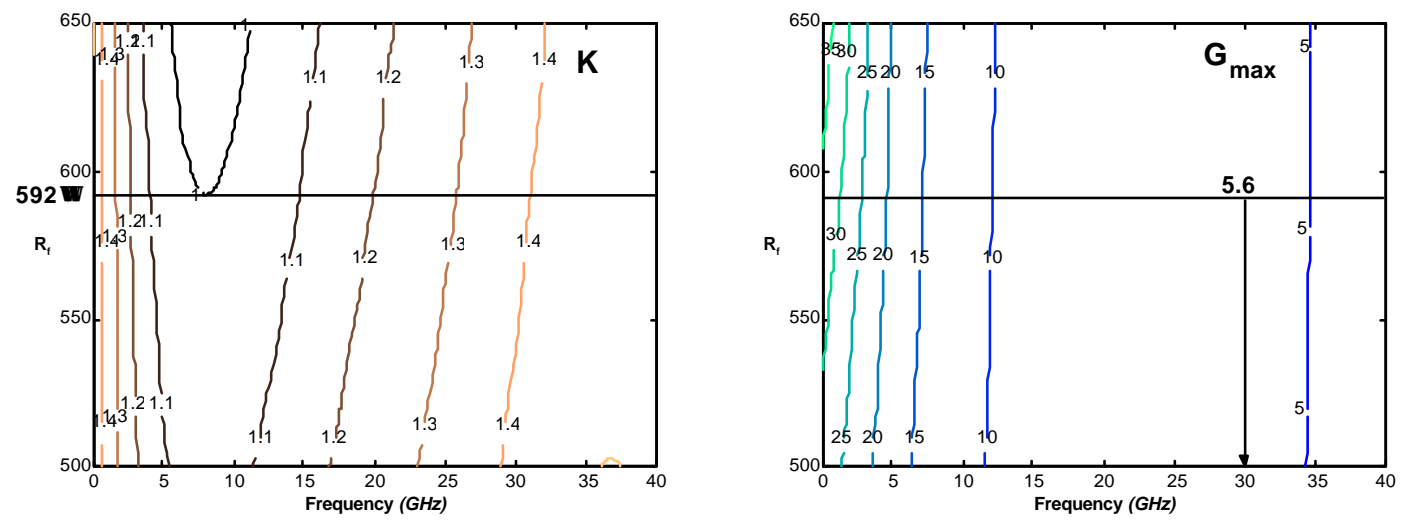

Figure 2 The $K$ and $G_{\max }$ contours for the $C S$ configuration for $L_{f}=0.1 \mathrm{nH}$ 

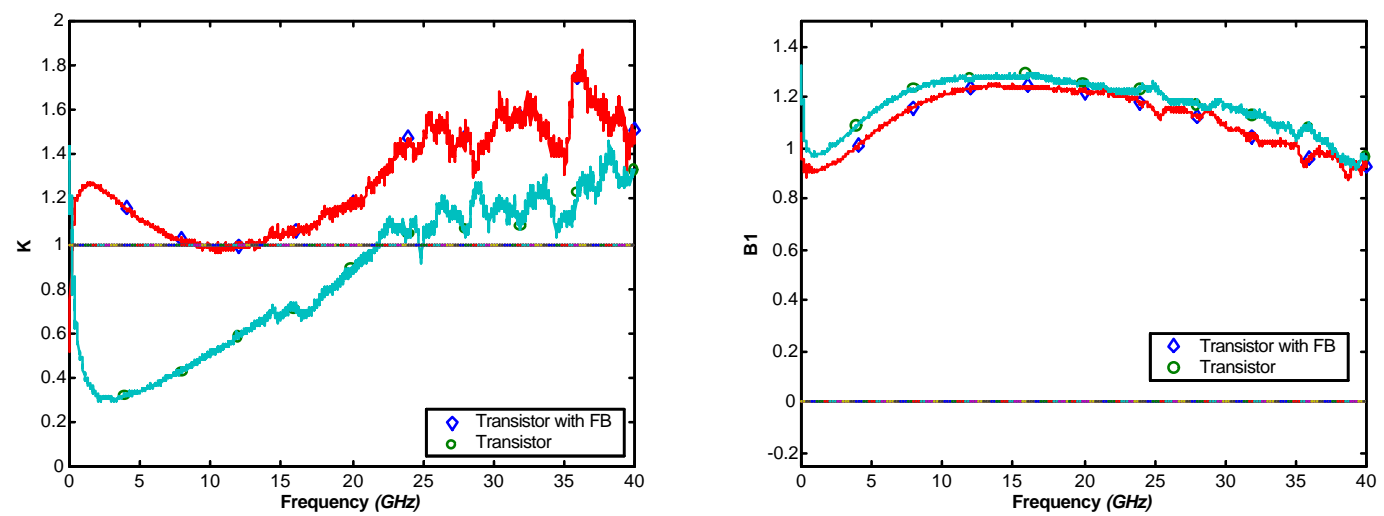

Figure 3 the measured value of $K$ and $B 1$ of the transistor with and without the stabilization network designed in Fig. (2)

\section{CONCLUSIONS}

A new technique for unconditionally sabilizing the MESFET transistor over a very wide range of frequency was established. The technique was applied to both the common source and the rarely used common gate transistor configurations. The feedback network offered wide frequency range of stabilization without critically reducing the maximum available stable gain. Analytical formulas were evaluated and they provide insight into the transistor stability performance. Several transistors were designed, fabricated and measured for different feedback values. The measured results showed very close agreement with the theoretical results.

\section{REFERENCES}

[1] G. Gonzalez, "Microwave transistor amplifiers: Analysis and design", $2^{\text {nd }}$ Edition, Prentice Hall, 1997.

[2] F. Perez, and V. Ortega, "A graphical method of the design of feedback networks for microwave transistor amplifiers: Theory and Applications", IEEE Trans. MTT, Oct. 1981, vol. MTT-29, No. 10, pp 1019-1027.

[3] K.B. Niclas, W.T. Wilser, R.B. Gold and W.R. Hitchens, "The matched feedback amplifier: ultrawideband microwave amplification with GaAs MESFET's", IEEE Trans. MTT, April 1980, Vol. MTT-28, No. 4, pp 285- 294

[4] K. B. Niclas, and W.T. Wilser, "A 2-12 GHz feedback amplifier on GaAs", MTT-S, 1981, pp 356-358 